\title{
Burnout e fatores associados entre profissionais de enfermagem de hospital
}

\section{municipal}

\author{
Burnout and associated factors among nursing professionals at a municipal hospital \\ Burnout y factores asociados entre los profesionales de enfermería de un hospital municipal
}

Recebido: 12/02/2021 | Revisado: 20/02/2021 | Aceito: 23/02/2021 | Publicado: 02/03/2021

\author{
Rafael da Silva Soares \\ ORCID: https://orcid.org/0000-0002-0796-0835 \\ Universidade Federal Fluminense, Brasil \\ E-mail: rafaelsoares@id.uff.br \\ Enéas Rangel Teixeira \\ ORCID: https://orcid.org/0000-0002-1721-2056 \\ Universidade Federal Fluminense, Brasil \\ E-mail: eneaspsi@hotmail.com \\ Jorge Luiz Lima da Silva \\ ORCID: https://orcid.org/0000-0002-2370-6343 \\ Universidade Federal Fluminense, Brasil \\ E-mail: jorgeluizlima@gmail.com \\ Marléa Crescêncio Chagas \\ ORCID: https://orcid.org/0000-0002-6122-7300 \\ Universidade Federal do Rio de Janeiro, Brasil \\ E-mail: marleachagas@gmail.com
}

\begin{abstract}
Resumo
Objetivo: descrever a prevalência e possíveis fatores associados à síndrome de burnout entre profissionais de enfermagem de unidade de terapia intensiva. Metodologia: estudo epidemiológico observacional, descritivo, seccional. A população foi composta por amostra de 85 profissionais de enfermagem dos setores de terapia intensiva de hospital público municipal. Foi utilizada a versão traduzida e validada para o português do Maslach Burnout Inventory. Foi realizada análise estatística descritiva com medidas de tendência central, de dispersão e análise de frequência. A coleta de dados ocorreu em janeiro de 2018. Resultados: a prevalência de suspeição da síndrome de burnout foi de $40 \%$, sendo que $24,7 \%$ apresentaram esgotamento emocional alto, 18,8\% despersonificação elevada, e $08,2 \%$ realização profissional baixa. Ter cursado ensino superior $(p=0,028)$, profissional enfermeiro $(p=0,009)$ e sentir-se estressado no trabalho $(\mathrm{p}=0,001)$, apresentaram associação com a síndrome. Conclusão: A síndrome de burnout pode ser desencadeada por diferentes fatores: ambiente de trabalho; organizacionais, personalidade, relacionamentos. É necessário adotar medidas preventivas e controle de doenças relacionadas ao estresse laboral.

Palavras-chave: Estresse psicológico; Esgotamento profissional; Equipe de enfermagem; Unidades de terapia intensiva; Saúde do trabalhador.
\end{abstract}

\begin{abstract}
Aim: to describe the prevalence of suspicion and possible factors associated with burnout syndrome among critical care nursing professionals. Methodology: observational, descriptive, sectional epidemiological study. The population consisted of a sample of 85 nursing professionals from the intensive care sectors of a municipal public hospital. The translated and validated Portuguese version of the Maslach Burnout Inventory was used. Descriptive statistical analysis was performed with measures of central tendency, dispersion and frequency analysis. Data collection took place in January 2018. Results: the prevalence of suspected burnout syndrome was $40 \%$, with $24.7 \%$ showing high emotional exhaustion, $18.8 \%$ high depersonification, and $08.2 \%$ low professional achievement. Having attended higher education $(p=0.028)$, a professional nurse $(p=0.009)$ and feeling stressed at work $(p=0.001)$, were associated with the syndrome. Conclusion: This syndrome can be triggered by different factors: work environment; organizational, personality, relationships. It is necessary to adopt preventive measures and control of diseases related to occupational stress.
\end{abstract}

Keywords: Psychological stress; Burnout professional; Nursing team; Intensive care units; Occupational health.

\section{Resumen}

Objetivo: describir la prevalencia de sospecha y posibles factores asociados al síndrome de burnout entre profesionales de enfermería de cuidados críticos. Metodología: estudio epidemiológico observacional, descriptivo, seccional. La población estuvo conformada por una muestra de 85 profesionales de enfermería de los sectores de cuidados intensivos de un hospital público municipal. Se utilizó la versión portuguesa traducida y validada del 
Maslach Burnout Inventory. Se realizó análisis estadístico descriptivo con medidas de análisis de tendencia central, dispersión y frecuencia. La recolección de datos tuvo lugar en enero de 2018. Resultados: la prevalencia de sospecha de síndrome de burnout fue del $40 \%$, con un $24,7 \%$ con alto agotamiento emocional, $18,8 \%$ con alta despersonalización y $08,2 \%$ con bajo rendimiento profesional. Haber cursado estudios superiores $(p=0,028)$, una enfermera profesional $(\mathrm{p}=0,009)$ y sentirse estresado en el trabajo $(\mathrm{p}=0,001)$, se asociaron con el síndrome. Conclusión: este síndrome puede ser desencadenado por diferentes factores: ambiente laboral; organizacional, personalidad, relaciones. Es necesario adoptar medidas preventivas y de control de enfermedades relacionadas con el estrés laboral.

Palabras clave: Estrés psicológico; Agotamiento profesional; Equipo de enfermería; Unidades de cuidados intensivos; Salud del trabajador.

\section{Introdução}

Por ser atividade de caráter social, o trabalho desempenha papel fundamental nas condições de vida dos indivíduos. Pelo trabalho são conferidas as oportunidades sociais de realização da cidadania (Araújo, 2017). Gera efeito positivo, quando consegue satisfazer as necessidades básicas de sustento, colaboração dos trabalhadores e de criação. Porém, o profissional sofre a exposição a variados agentes e fatores no ambiente ocupacional, podendo trazer interferência nas condições de saúde.

O incremento do atual modelo econômico produtivo produz novas vulnerabilidades sociais, ambientais e sanitárias ameaçando os sistemas que dão suporte à vida, alterando comunidades e grupos humanos, de modo que é preciso colocar em análise esse fenômeno, e levantar questões do ponto de vista da saúde pública, especificamente da vigilância em saúde, trabalho e ambiente (Leão \& Vasconcellos, 2015).

Nesse cenário, com desenvolvimento da economia e das tecnologias, o contexto organizacional e estrutural, as formas de adoecer e morrer dos trabalhadores estão se remodelando, o que exprime o crescimento de doenças como fadiga física e mental e outras manifestações de sofrimento relacionadas ao trabalho (Camelo et al. 2014; Trindade, Merlo, Silva, Beck \& Goes, 2017), com repercussão na saúde mental (Silva, Lira, Feijão, Costa \& Medeiros, 2017).

Neste sentido, o trabalhador utiliza menos tempo para cuidar de sua própria saúde, dedicando-se excessivamente ao trabalho; para que, dessa forma, consiga manter-se participante e ativo num contexto cada vez mais exigente e competitivo, mesmo com baixa remuneração. Desse modo, amplia sua carga horária, até mesmo com outros vínculos empregatícios para manter o padrão de vida (Palma, Suazo \& Alvarado, 2015).

A enfermagem possui jornadas de trabalho na maioria das vezes exaustiva, devido ao grande volume de pacientes e o pouco tempo de descanso. Assim, possuem seus padrões de sono, alimentação e atividades sociais alterados, principalmente em plantões noturnos. E, muitas vezes, esses se dedicam a mais de um emprego, devido aos salários não compatíveis com a realidade social (Rodrigues et al. 2017). Os problemas ocasionados por esses fatos tornam-se evidentes. São obtidos pela labuta diária associada ao estilo de vida dos profissionais e, ao terem interferência na qualidade de vida, adquirem mudanças nas atitudes. Pode-se perceber que esses fatores exercem impactos tanto biológicos, quanto comportamental e profissional nos indivíduos.

A síndrome de burnout (SB) é definida como estresse crônico experimentado pelo indivíduo em seu contexto de trabalho, principalmente no âmbito das profissões cuja característica essencial é o contato direto com pessoas como, por exemplo, professores (Koga et al. 2015), médicos (Tironi et al. 2016) e profissionais de enfermagem (Silva et al. 2015).

É avaliada por três dimensões: exaustão emocional (EE), despersonificação (DP) e realização profissional (RP). A dimensão exaustão emocional refere-se a sentimento de fadiga e esgotamento energético, que esvazia os recursos emocionais do indivíduo. Despersonificação, componente interpessoal do burnout, engloba as atitudes negativas de dureza, indiferença e distanciamento excessivo, manifestas pelos profissionais no relacionamento com os usuários dos seus serviços. A dimensão realização profissional, quando baixa, relaciona-se a sentimento de incompetência e a percepção de desempenho insatisfatório no trabalho (Maslach \& Jackson, 1981). 
A SB está relacionada ao esgotamento energético, expresso por meio de sentimento de fracasso e exaustão, causados por excessivo desgaste de recursos de enfrentamento. As recentes visões sobre a SB a explicam a partir de novas interpretações da noção de desajuste pessoa-trabalho. Seis tipos de desajuste pessoa-trabalho são considerados de grande importância para o desencadeamento da síndrome: sobrecarga de trabalho, falta de controle, falta de recompensas, falta de senso comunitário, falta de justiça e conflitos de valor (Boechat \& Ferreira, 2014).

A atividade laboral hospitalar é caracterizada por excessiva carga de trabalho, contato com situações limitantes, altos níveis de tensão e riscos. Devido às próprias características do trabalho, as equipes de enfermagem e médica são mais suscetíveis ao estresse ocupacional (Silva et al. 2015).

Trabalhadores de enfermagem enfrentam mais sofrimento psicológico do que a população em geral, devido à exposição a vários elementos geradores de desgaste (Azevedo, Nery \& Cardoso, 2017). Há constante contato com doenças, exposição a fatores de risco de natureza física, química, biológica e psíquica. A complexidade dos inúmeros procedimentos realizados pela equipe, o grau de responsabilidade nas tomadas de decisão, a falta de recursos humanos, os possíveis acidentes de trabalho e o trabalho por turnos aumentam a angústia e a ansiedade desses profissionais, desencadeando situações de estresse e burnout (Rissardo \& Gasparino, 2013).

Dentre os diversos setores no ambiente hospitalar, os setores fechados, como as unidades de terapia intensiva (UTI), apresentam alguns fatores que permitem maior exposição do profissional de enfermagem a situações estressoras. Os setores fechados do hospital são unidades onda há reduzida interação com os demais setores, e é onde se encontram os pacientes internados que mais necessitam de cuidados diretos e intensivos, pois correm risco iminente, assim tem-se como exemplos as UTI de adultos, pediátrica e neonatal, e a unidade coronariana (UCO). A morte é fato intrínseco dessas unidades e requer rigoroso controle emocional da equipe com seus pacientes e familiares. Portanto, há a exigência de habilidades e extrema atenção dos profissionais, não só técnica, pois, além de tudo, são muitos pacientes na mesma situação (Cruz \& Abellan, 2015).

A SB pode predispor os profissionais de enfermagem a piores condições de saúde, favorecendo ao círculo vicioso, o que pode levar à má-qualidade da assistência ao paciente e aumento dos gastos organizacionais. Tendo em vista a dinâmica vivenciada por esses profissionais, se desperta a necessidade de pesquisa da temática para estabelecer meios de intervenção e posterior prevenção.

Dessa forma, o objetivo do trabalho é descrever a prevalência e possíveis fatores associados à síndrome de burnout entre profissionais de enfermagem de unidade de terapia intensiva.

\section{Metodologia}

A pesquisa se deu por meio de estudo epidemiológico observacional, descritivo seccional. A coleta de dados ocorreu em janeiro de 2018. O cenário foi hospital público municipal de emergência, de grande porte, localizado em região central no município do Rio de Janeiro. Conta com três setores de terapia intensiva, sendo duas unidades de terapia intensiva gerais, UTI(A) e UTI(B), e uma unidade coronariana, UCO. A população total contava com 107 profissionais da equipe de enfermagem desses setores. Optou-se pela realização de cálculo amostral, cuja fórmula se encontra na figura abaixo (Figura 1), que contou com a participação de 85 profissionais de enfermagem. 
Figura 1. Cálculo amostral.

$$
n=\frac{N \cdot Z^{2} \cdot p \cdot(1-p)}{Z^{2} \cdot p \cdot(1-p)+e^{2} \cdot(N-1)}
$$

Legenda: $\mathrm{n}$ - amostra calculada; N - população; Z - variável normal padronizada associada ao nível de confiança; p - verdadeira probabilidade do evento; e - erro amostral. Fonte: Autores (2020), com base na fórmula de cálculo amostral (Santos, 2011).

Foram incluídos como participantes da pesquisa: 20 enfermeiros (23,5\%), e 65 técnicos e auxiliares de enfermagem (76,5\%), sem diferenciação de tipo de vínculo ou turno, credo ou religião, de ambos os sexos, sendo requerida ainda a assinatura do termo de consentimento. Aqueles que atuavam a menos de doze meses nos setores de terapia intensiva não foram incluídos na pesquisa.

O instrumento utilizado foi questionário estruturado com perguntas fechadas, elaborado pelos pesquisadores, contendo as seguintes seções: aspectos laborais; sociodemográficos; saúde física e emocional; antecedentes e hábitos de vida; e saúde e bem-estar. Este instrumento foi aplicado por profissional treinado e capacitado, tendo sido realizados teste e reteste para aplicação de tal instrumento na coleta de dados.

Para a para mensuração da síndrome de Burnout foi aplicada a versão traduzida e validada para o português do Maslach Burnout Inventory (MBI) (Tamayo, 1997). O instrumento é composto por 22 questões (Maslach \& Jackson, 1981). O MBI é formado por escala de frequência com cinco pontos que vai de um até cinco, que avaliam três dimensões: exaustão emocional (09 afirmativas), despersonificação (05 afirmativas) e realização profissional (08 afirmativas). A pontuação foi obtida pela soma dos valores em cada subescala (Benevides-Pereira, 2002).

O estudo foi aprovado por dois comitês de ética em pesquisa, da Universidade Federal Fluminense e Secretaria Municipal de Saúde do Rio de Janeiro.

Inicialmente, foi realizada análise univariada que permitiu descrever as características sociodemográficas, laborais e de estilo de vida da população estudada. As variáveis contínuas foram apresentadas, segundo suas frequências médias (com o respectivo desvio padrão) e as variáveis categóricas, segundo seus valores absolutos e proporções.

Para a análise bivariada, a variável idade foi analisada, segundo a média encontrada para população e em dois estratos. A escolaridade informada foi agrupada em duas categorias para análise univariada e bivariada: até ensino superior incompleto e acima de ensino superior. A renda foi analisada de acordo com os valores per capita, em salários mínimos da época em que foi realizada a coleta de dados ( $\mathrm{R}$ \$ 937,00), também foi avaliada, segundo a média encontrada. Para análise da situação conjugal, foram agrupadas duas categorias, a dos casados e a dos solteiros, divorciados, separados ou viúvos, denominadas neste estudo como: com companheiro (a) e sem companheiro (a), respectivamente.

O teste qui quadrado (x2) foi utilizado para verificar diferenças entre os grupos analisados durante a análise bivariada. Foi considerado, na avaliação da significância, o valor $\mathrm{p} \leq 0,05$.

A partir das análises realizadas foram desenvolvidas 3 categorias a serem discutidas, sendo elas: possível risco à saúde mental e qualidade de vida do trabalhador; a despersonificacão e o afastamento do cuidado; possível comprometimento na qualidade do cuidado.

\section{Resultados}

Participaram da pesquisa 85 profissionais de enfermagem. Quanto ao perfil sociodemográfico da população, média de idade de 48 anos (DP \pm 9,7 anos), com 47 indivíduos acima da média (55,3\%); em relação ao sexo, 62 mulheres (72,9\%); 
quanto à cor de pele, 39 mestiços (45,9\%), 27 pretos (31,8\%) e 19 brancos (22,3\%); em relação à presença de companheiro (a), 52 possuem (61,2\%); quanto à presença de filhos, 60 possuem (70,6\%); no quesito renda familiar, a média ficou entre 4 e 5 salários mínimos, com 66 profissionais acima dessa média (77,6\%); quanto à escolaridade, 53 possuem ensino superior completo $(62,4 \%)$.

Quanto a aspectos de vida e saúde, a maioria da população é não fumante 74 (87,1\%), não etilista 64 (75,3\%), dedica tempo ao lazer $59(69,4 \%)$, e não pratica atividades físicas regularmente 54 (63,3\%). Em relação a morbidades, 51 (60,0\%) não possuem hipertensão arterial sistêmica, e 78 (91,8\%) não são diabéticos.

Quanto à categoria profissional, há 65 técnicos/auxiliares de enfermagem (76,5\%) e 20 enfermeiros (23,5\%); em relação ao contrato de trabalho, 84 são estatutários (98,8\%), distribuídos nos seguintes setores: UTI(A) 37 (43,5\%), UCO 26 (30,6\%), e UTI(B) $22(25,9 \%)$.

Em relação a outros empregos, 53 (62,4\%) os possuem, perfazendo carga horária semanal total média de 53 horas (DP $\pm 20,7$ horas), com $51(60,0 \%)$ trabalhadores acima dessa média. Quanto ao tempo trabalhado na instituição, a média é de 17 anos (DP $\pm 8,7$ anos), com 50 (58,8\%) funcionários com tempo inferior à média; quanto ao tempo no setor, a média é de 12 anos (DP $\pm 7,4$ anos), com 44 (51,8\%) funcionários com tempo inferior à média.

Em relação ao turno de trabalho, 42 (49,4\%) são plantonistas noturnos, 37 (43,5\%) plantonistas diurnos e $6(7,1 \%)$ diaristas, com média de permanência no turno em 13 anos (DP $\pm 7,6$ anos), sendo $47(55,3 \%)$ com tempo superior à média.

A prevalência global da síndrome de burnout encontrada foi de $40 \%$. Considerando-se os pontos de cortes estabelecidos para cada dimensão: esgotamento emocional (alto, acima de 27 pontos): 21 (24,7\%) suspeitos; despersonificação (alta, acima de 10 pontos): 16 (18,8\%) suspeitos; realização profissional (baixa, acima de 40 pontos): 07 (08,2\%) suspeitos Tabela 1.

Tabela 1. Prevalência de acordo com as dimensões da SB entre trabalhadores de enfermagem, de setores intensivos de hospital municipal - Rio de Janeiro.

\begin{tabular}{lccc}
\hline \multicolumn{1}{c}{ DIMENSÕES } & Alto n (\%) & Médio n (\%) & Baixo n (\%) \\
\hline Esgotamento emocional & $21(24,7 \%)$ & $12(14,1 \%)$ & $52(61,2 \%)$ \\
Despersonificação & $16(18,8 \%)$ & $11(12,9 \%)$ & $58(68,3 \%)$ \\
Realização profissional & $58(68,3 \%)$ & $20(23,5 \%)$ & $07(08,2 \%)$ \\
\hline
\end{tabular}

Legenda: $\mathrm{n}=$ número de trabalhadores suspeitos. \% = prevalência. Fonte: Autores (2020).

Quanto à confiabilidade interna geral da escala, o alfa de Cronbach foi de 0,783. Considerando individualmente cada dimensão: o alfa para EE foi de 0,923; para RP 0,822; e, para DP 0,683. Tais achados denotam confiabilidade interna do instrumento para avaliação de burnout.

Considerando o critério de diagnóstico de burnout idealizado por Grunfeld et al. (2000), a prevalência da síndrome atingiu $34(40,0 \%)$ trabalhadores de enfermagem, nas unidades de terapia intensiva. Sob o critério de Golembiewski, Munzenrider \& Carter (1983), a prevalência seria de 18,8\%, atingindo 16 profissionais de enfermagem. Pelo modelo de avaliação de Ramirez et al. (1996), há ausência de SB entre os pesquisados. 
Tabela 2. Prevalência de SB, segundo variáveis sociodemográficas, entre trabalhadores de enfermagem, de setores intensivos de hospital municipal - Rio de Janeiro.

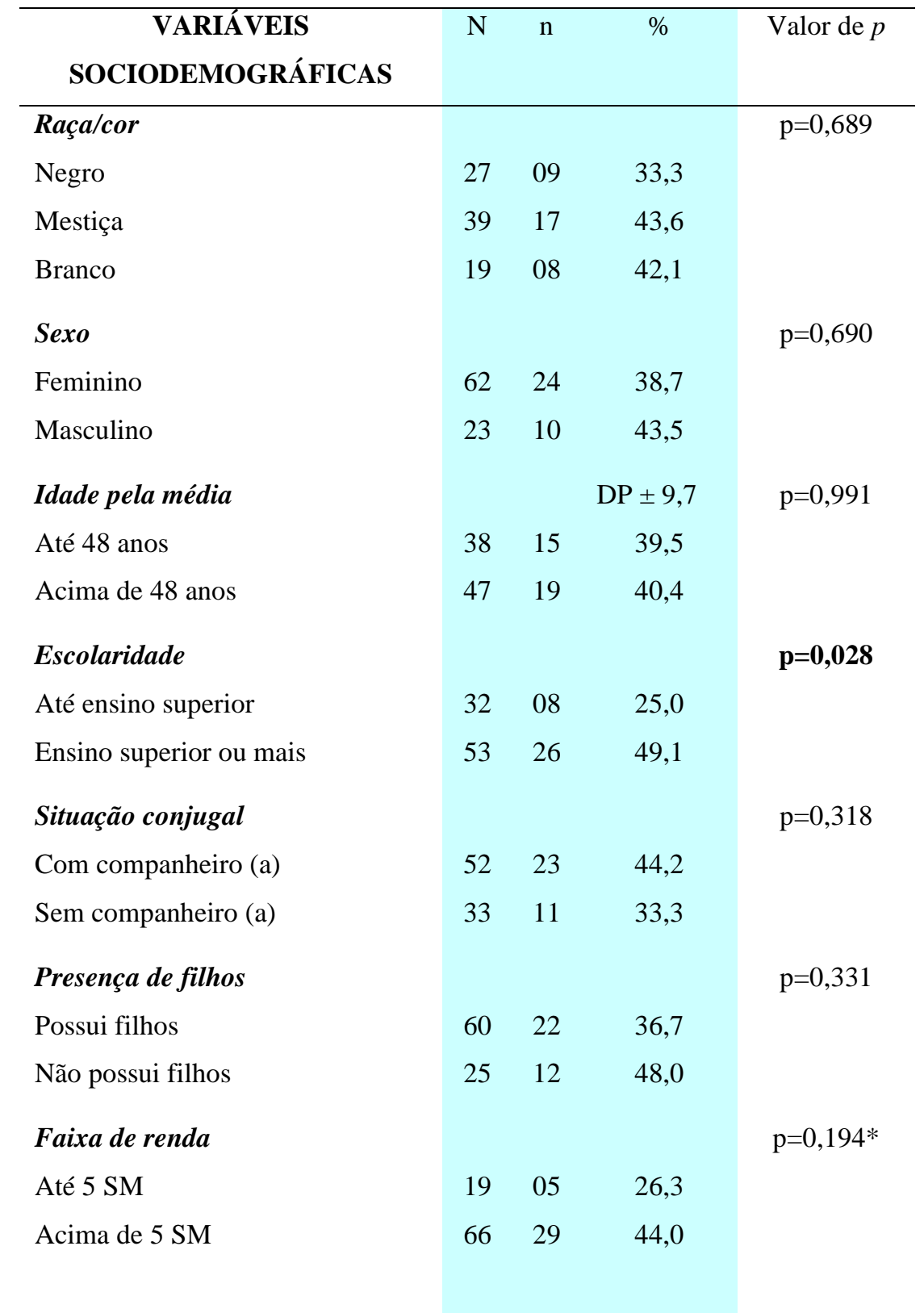

Legenda: $\mathrm{N}=$ total no estrato. $\mathrm{n}=$ número de trabalhadores suspeitos. \% = prevalência. ${ }^{*}$ Teste de Fisher. Fonte: Autores (2020).

Entre as variáveis sociodemográficas, apenas o grau de instrução esteve associado estatisticamente à suspeição da SB. Nesse caso, ter cursado ensino superior $(\mathrm{p}=0,028)$. 
Tabela 3. Prevalência de SB, segundo variáveis laborais, entre trabalhadores de enfermagem, de setores intensivos de hospital municipal - Rio de Janeiro.

\begin{tabular}{|c|c|c|c|c|}
\hline VARIÁVEIS LABORAIS & $\mathrm{N}$ & $\mathrm{n}$ & $\%$ & Valor de $\mathrm{p}$ \\
\hline Tempo no hospital & & & $\mathrm{DP} \pm 8,7$ & $\mathrm{p}=0,177$ \\
\hline Até 17 anos & 50 & 17 & 34,0 & \\
\hline Acima de 17 anos & 35 & 17 & 48,6 & \\
\hline Tempo no turno & & & $\mathrm{DP} \pm 7,6$ & \\
\hline Até 13 anos & 38 & 15 & 39,5 & $\mathrm{p}=0,929$ \\
\hline Acima de 13 anos & 47 & 19 & 40,4 & \\
\hline Tempo no setor & & & $\mathrm{DP} \pm 7,4$ & \\
\hline Até 12 anos & 44 & 18 & 40,9 & $\mathrm{p}=0,859$ \\
\hline Acima de 12 anos & 41 & 16 & 39,0 & \\
\hline Outros empregos & & & & $\mathrm{p}=0,715$ \\
\hline $\operatorname{Sim}$ & 53 & 22 & 41,5 & \\
\hline Não & 32 & 12 & 37,5 & \\
\hline Turno de trabalho & & & & $\mathrm{p}=0,850$ \\
\hline Diarista & 06 & 03 & 50,0 & \\
\hline Plantonista dia & 37 & 14 & 37,8 & \\
\hline Plantonista noite & 42 & 17 & 40,5 & \\
\hline Carga horária total & & & $\mathrm{DP} \pm 20,7$ & $\mathrm{p}=0,786$ \\
\hline Abaixo de $53 \mathrm{~h}$ & 34 & 13 & 38,2 & \\
\hline Acima de $53 \mathrm{~h}$ & 51 & 21 & 41,2 & \\
\hline Carga horária semanal & & & $\mathrm{DP} \pm 2,2$ & $\mathrm{p}=0,600^{*}$ \\
\hline Abaixo de $30 \mathrm{~h}$ & 01 & 00 & 00,0 & \\
\hline Acima de $30 \mathrm{~h}$ & 84 & 34 & 40,5 & \\
\hline Setores & & & & $\mathrm{p}=0,520$ \\
\hline $\mathrm{UTI}(\mathrm{A})$ & 37 & 13 & 35,1 & \\
\hline $\mathrm{UCO}$ & 26 & 10 & 38,5 & \\
\hline UTI(B) & 22 & 11 & 50,0 & \\
\hline Tipo de vínculo & & & & $\mathrm{p}=0,400 *$ \\
\hline Temporário & 01 & 01 & 100,0 & \\
\hline Permanente & 84 & 33 & 39,3 & \\
\hline Pensamento durante a folga & & & & $\mathrm{p}=0,064$ \\
\hline Concordo & 55 & 26 & 47,3 & \\
\hline Discordo & 30 & 08 & 26,7 & \\
\hline Estresse autorreferido & & & & $p=0,001$ \\
\hline Sem estresse & 66 & 20 & 30,3 & \\
\hline
\end{tabular}


Estressado

Mudança no ambiente

Sim

Não

Categoria profissional
1914

$48-19$

$48 \quad 19$

$37 \quad 15$
73.7

39,6

40,5

\section{Auxiliar ou Técnico}

Enfermeiro

$65 \quad 21$

$20 \quad 13$

32,3

65,0

$\mathrm{p}=0,929$

$\mathbf{p}=\mathbf{0 , 0 0 9}$

Legenda: $\mathrm{N}=$ total no estrato. $\mathrm{n}=$ número de trabalhadores suspeitos. $\%=$ prevalência. ${ }^{*}$ Teste de Fisher. Fonte: Autores (2020).

Quanto às variáveis relacionadas ao trabalho, a categoria profissional enfermeiro $(\mathrm{p}=0,009)$, e sentir-se estressado $(\mathrm{p}=0,001)$ demonstraram associação à suspeição da SB.

Entre as variáveis relacionadas à saúde, não foram observadas associações estatísticas com a suspeição de SB.

\section{Discussão}

Neste estudo, considerando-se os pontos de cortes estabelecidos para cada dimensão da síndrome de burnout, foi constatado que o esgotamento emocional alto afeta 21 (24,7\%) profissionais. A despersonificação alta atingiu, naquele dado momento, 16 trabalhadores $(18,8 \%)$, e a realização profissional baixa $7(8,2 \%)$ indivíduos.

O grau de exaustão emocional elevado ocorreu para 21,3\% (Gallegos \& Toia, 2016). Pesquisa desenvolvida na Espanha identificou altos valores de EE entre 25,0\% dos profissionais de enfermagem (Cañadas-Dela Fuente et al. 2015), valores próximos ao deste estudo.

Estudo evidenciou alto grau de DP entre 16,8\% da equipe de enfermagem (Escarón \& Balado, 2016). Outra pesquisa identificou 21,9\% dos profissionais de enfermagem com alto padrão de despersonificação (Ferreira \& Lucca, 2015), valores semelhantes aos resultados encontrados, neste estudo.

Quanto à baixa RP, em pesquisa desenvolvida no Uruguai, foi encontrado valor de 9,6\% entre profissionais de enfermagem (Escarón \& Balado, 2016). Em estudo realizado na Grécia foi de 12,2\% (Ntantana et al. 2017), valores um pouco mais elevados, em relação aos deste estudo.

Neste estudo, considerando o critério de diagnóstico de Burnout idealizado por Grunfeld et al. (2000), a prevalência global da síndrome atingiu 34 (40,0\%) trabalhadores. Utilizando os mesmos critérios, pesquisa identificou a prevalência de SB em 55,3\% dos profissionais de enfermagem atuantes em unidades de terapia intensiva (Silva et al. 2015).

Os pesquisadores tendem a expor resultados, de acordo com as dimensões da síndrome. Percebe-se que a definição de burnout baseada em três dimensões em desequilíbrio concomitantes, possivelmente conduz a subestimação da prevalência, o que vem de encontro à relevância da identificação precoce dos sinais da SB, devido ao seu caráter incidioso.

Quanto às variáveis relacionadas ao trabalho, sentir-se estressado guardou associação positiva à prevalência da SB $(\mathrm{p}=0,001)$. Sabe-se que por essas características serem subjetivas e autorreferidas acabam por estar associadas, uma vez que o desgaste da síndrome conduz à percepção exacerbada de estresse na vida cotidiana. Estudos com escalas de avaliação de estresse têm mostrado associação do estresse no trabalho com esgotamento profissional (Lin, Lin, Cheng, Wu \& Ou-Yang, 2016; Orgambídez-Ramos, Borrego-Alés \& Ruiz-Frutos, 2018).

Quando as pessoas parecem ser sensíveis ao estresse, podem sentir-se excessivamente saturados quando enfrentam demandas laborais cotidianas que seus colegas podem considerar normais. A vulnerabilidade afetaria as estratégias de enfrentamento dos profissionais, criando expectativas negativas sobre seu desempenho e sobre o ambiente de trabalho. Se os 
profissionais de enfermagem reagirem exageradamente (alta vulnerabilidade), quando as demandas estressantes aparecem no trabalho, suas habilidades de enfrentamento e autoeficácia são reduzidas, afetando seu desempenho e criando um círculo vicioso: mais estresse, pior enfrentamento, pior desempenho e maior estresse. Se esses níveis de estresse se tornarem frequentes, é muito provável que a síndrome surja (Orgambídez-Ramos, Borrego-Alés \& Ruiz-Frutos, 2018).

Entre as variáveis sociodemográficas, o grau de instrução esteve associado à prevalência da SB, ensino superior ( $\mathrm{p}=0,028)$, assim como a categoria "enfermeiro" $(\mathrm{p}=0,009)$. Pesquisas demonstram que as pessoas com maior nível de escolaridade possuem maior probabilidade de desenvolver a SB (França \& Ferrari, 2012; Lima, 2016).

Em profissionais de enfermagem de hospital no Mato Grosso também foi evidenciado que em relação à escolaridade, houve predominância da síndrome nos profissionais com ensino superior completo (França \& Ferrari, 2012). No entanto, em trabalhadores de enfermagem de UTI de São Paulo, identificou-se que a maioria dos profissionais que havia concluído o ensino médio apresentou SB. Tais achados conduzem a questionar se o fato de pertencer à classe profissional subordinada, em que há exigências e demandas, com salários mais baixos, pode influenciar no desenvolvimento da síndrome (Fernandes, Nitsche \& Godoy, 2017).

Contudo, indivíduos com maior grau de escolaridade são mais propensos ao desenvolvimento da SB, quando comparado àqueles com menor nível. É possível que aqueles com maior escolaridade possuam maiores expectativas, quanto ao seu sucesso profissional, além de responsabilidade sobre os demais profissionais de enfermagem de nível médio/técnico (Lima, 2016). Pois, os enfermeiros apresentam intensa sobrecarga emocional em seu cotidiano de trabalho, uma vez que são responsáveis por cuidados diretos ao doente grave, assim como pelo bom funcionamento do setor. Soma-se a supervisão dos demais profissionais de enfermagem, as demandas constantes das chefias, administração hospitalar, e outros profissionais da equipe de saúde nas UTI, como médicos e fisioterapeutas. Logo, quanto maior o nível educacional maior é a propensão à SB.

\section{Possível risco à saúde mental e qualidade de vida do trabalhador}

A depressão influi no desempenho profissional do das enfermeiras. Pesquisa em Taiwan revelou que os níveis de burnout e depressão ocupacional também foram correlacionados positivamente. Em nível organizacional, a depressão aumenta as taxas de rotatividade de enfermagem; e, indiretamente, aumenta a carga de trabalho de outras enfermeiras. Ao longo do tempo, é formado ciclo periódico de baixa satisfação no trabalho, alta rotatividade e recursos humanos insuficientes (Lin, Lin, Cheng, Wu \& Ou-Yang, 2016).

Foi verificada a correlação entre os escores das dimensões do burnout e o escore total de sintomatologia depressiva, pode-se dizer que existiu correlação, em que, quanto maior foi o nível de exaustão emocional e de despersonificação, maior a sintomatologia depressiva; e, quanto menor a realização profissional (inversamente proporcional), maior a sintomatologia depressiva (Vasconcelos, Martino \& França, 2018).

Pesquisas no Brasil, Estados Unidos e Espanha revelaram impacto do burnout na qualidade do sono (Andolhe, Barbosa, Oliveira, Costa \& Padilha, 2015; Sánchez, Martínez, Sahuquillo, Román \& Cantó, 2017). A privação do sono provoca diversos distúrbios, sendo os principais: gastrointestinais; cardiovasculares; cognitivos; flutuações de humor; comprometimento do desempenho das atividades; pessoais; sociais e do trabalho, pois diminui a capacidade de concentração e atenção. Assim, as horas de sono adequadas para a restauração do organismo desgastado pela jornada de trabalho parecem contribuir para evitar os sinais e sintomas de desgaste físico, sobretudo, do estresse e burnout, citados anteriormente, e são de extrema importância para a saúde do indivíduo (Andolhe, Barbosa, Oliveira, Costa \& Padilha, 2015).

Quanto às dimensões do burnout em relação a eventos de vida estressantes, evidenciou-se que os profissionais que experimentaram mudança nas condições de trabalho têm maior esgotamento emocional. Ocorre do mesmo modo naqueles que tiveram a morte de parente próximo, mudanças na situação econômica, nas atividades sociais e nos hábitos alimentares 
(Sánchez, Martínez, Sahuquillo, Román \& Cantó, 2017).

A insônia, falta de descanso, nervosismo e cansaço foram as queixas mais comuns dos profissionais. A análise revelou que apenas o cansaço pode ser considerado sintoma associado ao burnout, todos os outros sintomas não tiveram associação estatística (Skorobogatova, Žemaitienè, Šmigelskas \& Tamelienè, 2017).

A situação econômica afetou os profissionais de enfermagem na Espanha, de modo que, a partir de 2010, muitas administrações aproveitam a situação para reduzir os recursos humanos e materiais como medida imediata de poupança, gerando sobrecarga de trabalho e piora dos cuidados prestados. Esta medida, adicionada à redução ou congelamento de salários, aumento da carga horária, diminuição de pessoal, deteriorou o ambiente de trabalho e provocou desmotivação em nível profissional. A síndrome de burnout foi ligeiramente superior ao período pré-crise. O esgotamento emocional foi a dimensão mais influente no aparecimento da SB, desde o início da crise econômica (Sánchez, Martínez, Sahuquillo, Román \& Cantó, 2017).

\section{A despersonificacão e o afastamento do cuidado}

A despersonificação manifesta-se por atitude cínica e distante, em relação aos pacientes, que tem sido interpretada também como mecanismo de proteção. Isto é, dado que a síndrome é influenciada pelo contato com os pacientes, com isso, uma forma de reduzir o mal-estar é não se comprometer com seus problemas e necessidades.

Pesquisa peruana correlacionou negativamente a despersonificação com baixa realização profissional, ou seja, quanto maior a despersonificação, menor realização profissional. Nesse sentido, a falta de contato dos enfermeiros com a realidade, constitui característica da despersonificação, manifestada pela falta de interesse pelo paciente, e consequente afastamento do trabalho que é realizado (Gallegos \& Toia, 2016). Com isso, esse afastamento, mostra-se como "descuidado" aos pacientes; e, consequentemente, pode afetar a realização trabalhadores, em suas atividades diárias de cuidado às pessoas. Nesse aspecto, a síndrome de burnout influencia negativamente dois aspectos da PNH (Brasil, 2004): a assistência e a valorização profissional. Ou seja, a despersonificação afasta o profissional de enfermagem do cuidado. E a baixa realização profissional atrapalha a articulação entre os trabalhadores, impedindo seu crescimento e satisfação profissional.

\section{Possível comprometimento na qualidade do cuidado}

Naturalmente, o trabalhador de enfermagem afetado pela síndrome de burnout não produzirá cuidado de qualidade, haja vista sua exaustão emocional, que aumenta o desgaste físico e emocional; a diminuição da realização profissional, perdendo o sentimento de recompensa por seu cuidado prestado; e a despersonificação, que pode levar o profissional a ficar emocionalmente mais rígido e tratar pessoas como objetos, afastando-o do cuidado.

Pesquisa identificou que existe relação positiva e direta entre autonomia e burnout. Quanto maior o senso de autonomia, maior a percepção de realização pessoal, uma vez que o maior senso de autonomia pode ter efeito protetor no desenvolvimento do burnout, logo essa percepção tem efeito protetor para a baixa realização pessoal (Nogueira et al. 2018). A liberdade do enfermeiro para resolver problemas que afetam a qualidade da assistência de enfermagem acaba por favorecer ao trabalhador e a qualidade do cuidado.

A relação entre as dimensões de burnout e as variáveis de trabalho mostra que o esgotamento emocional é maior entre aqueles que percebem o ambiente de trabalho como ruim/muito ruim, em comparação com aqueles que o consideram regular, bom/muito bom. O mesmo acontece com a despersonificação. Em termos de realização pessoal, essa é maior naqueles que consideram seu ambiente de trabalho como bom/muito bom, em comparação com aqueles que o percebem como ruim/muito ruim, segundo estudo espanhol (Sánchez JM, Martínez, Sahuquillo, Román \& Cantó, 2017).

Estudo chinês identificou que o ambiente de trabalho influencia a satisfação profissional, que a satisfação profissional 
influencia o burnout, e que essa síndrome afeta negativamente a percepção das enfermeiras sobre o grau de excelência nos serviços de enfermagem (Liu \& Aungsuroch, 2017). Ou seja, tanto a percepção do ambiente de trabalho como ruim, como a insatisfação laboral, associam-se ao burnout. Isso quer dizer que o burnout influencia diretamente, na diminuição da qualidade do cuidado.

As demandas de trabalho e o burnout exibiram relação com presenteísmo entre enfermeiros. As demandas elevadas de trabalho causaram mais presenteísmo, assim como despersonificação, em longo do prazo (Demerout, Le Blanc, Bakker, Schaufeli \& Hox J, 2009; Brborović, Daka, Dakaj \& Brborović, 2017). Desse modo, o excesso de trabalho favorece o presenteísmo; que, por sua vez, limita a produtividade tanto em quantidade, quanto na qualidade do trabalho. Diminui o rendimento físico e mental do trabalhador, aumenta o sentimento de desgaste e frieza, bem como a possibilidade de causar prejuízos à atenção no desenvolvimento da assistência.

Identificou-se que o burnout diminui o compromisso profissional. O compromisso profissional foi definido como o grau em que um indivíduo é dedicado e orgulhoso de ser membro de uma profissão, e como acredita nos valores e objetivos da profissão (Chang et al. 2017). Isso significa que os profissionais de enfermagem com burnout desejam abandonar a instituição onde trabalham, mas não gostariam de desistir da enfermagem. Ou seja, buscam melhores condições de trabalho, mas, em outras organizações.

Profissionais com burnout têm mais intenção de abandonar o trabalho (Liu \& Aungsuroch, 2017). Com isso, forma-se círculo vicioso, no qual o trabalhador insatisfeito abandona o trabalho, deixando seus colegas em defasagem, até a chegada de novos profissionais naquele ambiente, para que se submetam as mesmas condições difíceis de trabalho.

\section{Conclusão}

A prevalência global da síndrome de burnout foi de $40 \%$.

A síndrome de burnout pode ser desencadeada por diferentes fatores, tais como ambiente de trabalho, fatores organizacionais, características pessoais, relacionamento interpessoal, deixando o profissional de enfermagem esgotado, frio e insatisfeito com seu trabalho, o que pode levar ao comprometimento da qualidade do cuidado prestado aos pacientes.

Além do mais, os prejuízos do burnout extrapolam a esfera profissional, podendo causar prejuízos na qualidade de vida, com os seguintes sintomas: depressão; distúrbios gastrointestinais; cardiovasculares; cognitivos; flutuações de humor; comprometimento do desempenho das atividades; diminuição da capacidade de concentração e atenção; sono prejudicado, entre outros.

Deste modo, são imperativos esforços em suprir as necessidades desses profissionais. Há necessidade de execução/efetivação das várias políticas públicas com mais eficácia e responsabilidade por parte das autoridades de saúde e dos empregadores. É necessário adotar medidas preventivas e de controle de doenças laborais.

Propõe-se intervenções em três aspectos: políticos, institucionais e de qualidade de vida no trabalho, para que haja mudança significativa para os trabalhadores, com aumento de seu bem-estar.

Por tratar-se de estudo epidemiológico transversal, não foi possível associar a exposição da doença e período de investigação, determinar risco absoluto e duração da doença.

\section{Agradecimentos}

O presente trabalho foi realizado com apoio da Coordenação de Aperfeiçoamento de Pessoal de Nível Superior Brasil (CAPES) - Código de Financiamento 001. 


\section{Referências}

Andolhe, R., Barbosa, R. L., Oliveira, E. M., Costa, A. L. S. \& Padilha, K. G. (2015). Stress, coping and burnout among Intensive Care Unit nursing staff: associated factors. Rev. Esc. Enferm. USP, 29(Sp): 58-64.

Araújo, J. M. (2017). Valor social do trabalho na Constituição Federal de 1988: instrumento de promoção de cidadania e de resistência à precarização. Rev. Direito Bras., 16(7): 115-34.

Azevedo, B. D. S., Nery, A. A. \& Cardoso, J. P. (2017). Occupational stress and dissatisfaction with quality of work life in nursing. Texto Contexto Enferm., 26(1).

Bamonti, P., Conti, E., Cavanagh, C., Gerolimatos, L., Gregg, J., Goulet, C. et al. (2017). Coping, cognitive emotion regulation, and burnout in long-term care nursing staff: a preliminary study. J. Appl. Gerontol, 38(1):92-111.

Benevides-Pereira, A. M. T. (2002). Burnout: quando o trabalho ameaça o bem-estar do trabalhador. Casa do Psicólogo.

Boechat, M. A. M. \& Ferreira, M. C. (2014). Preditores individuais e organizacionais do burnout em servidores públicos federais. Psicol. Saúde Doenças, 15(3): 738-50.

Brborović, H., Daka, Q., Dakaj, K., Brborović, O. (2017). Antecedents and associations of sickness presenteeism and sickness absenteeism in nurses: a systematic review. Int. J. Nurs. Pract., 23(6).

Camelo, S. H. H. \& Rocha F. L. R. (2014). Minimel, V. A., Santos, A. P. A., Garcia, A. B., Scozzafave, M. C. S. Trabalhador de saúde: formas de adoecimento e estratégias de promoção à saúde. Rev. Eletr. Gest. Saúde, 5(3): 2220-9.

Cañadas-Dela Fuente, G. A., Vargas, C. San Luis, C., García, I., Cañadas, G. R. \& De la Fuente, E. (2015). Risk factors and prevalence of burnout syndrome in the nursing profession. Int. J. Nurs. Stud., 52(1): 240-9.

Chang, H. Y., Shyu, Y. L., Wong, M. K., Chu, T. L., Lo, Y. Y. \& Teng, C. I. (2017). How does burnout impact the three components of nursing professional commitment? Scand. J. Caring Sci., 31(4): 1003-11.

Cruz, S. P. \& Abellan, M. V. (2015). Professional burnout, stress and job satisfaction of nursing staff at a university hospital. Rev. Latinoam. Enferm., 23(3): 543-52.

Demerouti, E., Le Blanc, P. M., Bakker, A. B., Schaufeli, W. B. \& Hox, J. (2009). Present but sick: A three-wave study on job demands, presenteeism and burnout. Career Dev. Int., 14(1): 50-68.

Escarón, M. R. \& Balado, L. (2016). Degreeof Burnout in nursingfacilitiesoperatingroom in a public hospital, Montevideo Uruguay. Rev. Urug. Enferm., 11(2): 61-77.

Fernandes, L. S., Nitsche, M. J. T. \& Godoy, I. (2017). Burnout syndrome in nursingprofessionalsfromanintensivecareunit. Rev. Pesqui. Cuid. Fundam, 9(2): $551-7$.

Ferreira, N. N. \& Lucca, S. R. (2015). Burnout syndrome in nursing assistants of a public hospital in the state of São Paulo. Rev. Bras. Epidemiol, 18(1): 6879.

França, F. M. \& Ferrari, R. (2012). Burnout syndrome and the socio-demographic aspects of nursing professionals. Acta Paul. Enferm, $25(5)$ : 743-8.

Gallego, W. L. A. \& Toia, A. M. C. (2016). Burnout syndrome in the nursing staff from Arequipa. Rev. Cubana Salud. Publ., 42(4): 559-74.

Golembiewski, R. T., Munzenrider, R. \& Carter, D. (19833). Phases of progressive burnout and their work site covariants: critical issues in OD research and praxis. J. Appl. Behav. Sci., 19(4):461-81.

Grunfeld, E., Whelan, T. J., Zitzelsberger, L., Willan, A. R., Montesanto, B. \& Evans, W. K. (2000). Cancer care workers in Ontario: prevalence of burnout, job stress and job satisfaction. CMAJ, 163(2):166-9.

Koga, G. C. K., Melanda, F. N., Santos, H. G., Sant'Anna, F. L., González, A. D., Mesas, E. A. et al. (2015). Factors associated with worse levels in the burnout scale in basic education teachers. Cad. Saúde Colet., 23(3): 268-75.

Leão, L. H. C \& Vasconcellos, L. C. F. (2015). Commodity chain and surveillance in health, work and the environment. Saúde Soc, $24(4)$ : 1232-43.

Lima, A. S. (2016). Prevalência e fatores associados à síndrome de burnout nos profissionais da saúde da atenção primária de Juiz de Fora. Dissertação de mestrado. Universidade Federal de Juiz de Fora, Juiz de Fora, Brasil.

Lin, T.C., Lin, H. S., Cheng, S. F., Wu, L. M. \& Ou-Yang, M. C. (2016). Work stress, occupational burnout and depression levels: a clinical study of paediatric intensive care unit nurses in Taiwan. J. Clin. Nurs, 25(7-8): 1120-30.

Liu, Y. \& Aungsuroch, Y. (2017). Factors influencing nurse-assessed quality nursing care: a cross-sectional study in hospitals. J. Adv. Nurs. 74(4):935-945.

Maslach, C. \& Jackson, S. E. (1981).The measurement of experienced burnout. J. Occup. Behav, 2(2): 99-113.

Ministério da Saúde, Brasil. (2004). Humaniza SUS: Política Nacional de Humanização: a humanização como eixo norteador das práticas de atenção e gestão em todas as instâncias do SUS. Brasília: Ministério da Saúde.

Nogueira, L. S., Sousa, R. M. C., Guedes, E. S., Santos, M. A., Turrini, R. N. T. \& Cruz, D. A. L. M. (2018). Burnout and nursing work environment in public health institutions. Rev. Bras. Enferm, 71(2): 358-65. 
Ntantana, A., Matamis, D., Savvidou, S., Giannakou, M., Gouva, M., Nakos, G. et al. (2017). Burnout and job satisfaction of intensive care personnel and the relationship with personality and religious traits: an observational, multicenter, cross-sectional study. Intensive Crit. Care Nurs, 41:11-17.

Orgambídez-Ramos, A., Borrego-Alés, Y. \& Ruiz-Frutos, C. (2018). Empowerment, stress vulnerability and burnout among Portuguese nursing staff. Cienc. $e$ Saúde Colet., 23(1): 259-66.

Palma, F. S., Suazo, S. V. \& Alvarado, O. S. (2015). El trabajo del profesional de enfermería: revisión de la literatura. Cienc. Enferm, 21(2): 11-20.

Ramirez, A. J., Graham, J., Richards, M. A., Cull, A. \& Gregory, W. M. (1996). Mental health of hospital consultants: the effects of stress and satisfaction at work. Lancet, 347(9003):724-8.

Rissardo, M. P. \& Gasparino, R. C. (2013). Emotional exhaustion in nurses of a public hospital. Esc. Anna Nery Rev. Enferm, 17 (1): $128-32$.

Rodrigues, C. C. F. M., Salvador, P. T. C. O., Assis, Y. M. S., Gomes, A. T. L., Bezerril, M. S. \& Santos, V. E. P. (2017). Stress among nursing team members. Rev. Enferm. UFPE, 11(2): 601-8.

Sánchez, J. M., Martínez, N. A., Sahuquillo, M. L., Román, A. C. \& Cantó, M. M. (2017). Analysis of impact of the economic crisis on the syndrom eof Burnout and resilience in nursing. Enferm. Glob, 16(2): 315-35.

Santos, G. E. O. (2011). Cálculo amostral: calculadora on-line. http://www.calculoamostral.vai.la/.

Silva, C. C. S., Lira, A. L. B. C., Feijão, A. R., Costa, I. K. F. \& Medeiros, S. M. (2017). Burnout and health technologies in the context of primary health care nursing. Esc. Anna Nery Rev. Enferm, 21(2).

Silva, J. L. L., Soares, R. S., Costa, F. S., Ramos, D. S., Lima, F. B. \& Teixeira, L. R. (2015). Psychosocial factors and prevalence of burnout syndrome among nursing workers in intensive care units. Rev. Bras. Ter. Intensiva, 27(2): 125-33.

Skorobogatova, N., Žemaitienė, N., Šmigelskas, K. \& Tamelienė, R. (2017). Professional burnout and concurrent health complaints in neonatal nursing. Open Med., 12: 328-34.

Tamayo, M. R. (1997). Relação entre a síndrome de burnout e os valores organizacionais no pessoal de enfermagem de dois hospitais públicos. Dissertação de mestrado. Universidade de Brasília, Brasília, Brasil.

Tironi, M. O. S., Teles, J. M. M., Barros, D. S., Vieira, D. F. B. V., Silva Filho, C. M., Martins Júnior, D. F. et al. (2016). Prevalence of burnout syndrome in intensivist doctors in five Brazilian capitals. Rev. Bras. Ter. Intensiva, 28(3): 270-7.

Trindade, L. R., Merlo, A. R. C., Silva, R. M., Beck, C. L. C. \& Goes, N. C. (2017). Factors of illnesses of health workers: integrative review. Cien. Cuid. Saúde, 16(4)

Vasconcelos, E. M., Martino, M. M. F. \& França, S. P. S. (2018). Burnout and depressive symptoms in intensive care nurses: relationship analysis. Rev. Bras. Enferm, 71(1): 147-53. 